\title{
Candela per Square Meter
}

National Cancer Institute

\section{Source}

National Cancer Institute. Candela per Square Meter. NCI Thesaurus. Code C122204.

A unit of luminous intensity expressed as one candela per square meter of area. 